\title{
Generating Informative Conclusions for Argumentative Texts
}

\author{
Shahbaz Syed $^{\dagger} \quad$ Khalid Al-Khatib $^{\dagger} \quad$ Milad Alshomary ${ }^{\ddagger}$ \\ Henning Wachsmuth ${ }^{\ddagger}$ Martin Potthast ${ }^{\dagger}$ \\ ${ }^{\dagger}$ Leipzig University ${ }^{\ddagger}$ Paderborn University \\ <shahbaz.syed@uni-leipzig.de>
}

\begin{abstract}
The purpose of an argumentative text is to support a certain conclusion. Yet, they are often omitted, expecting readers to infer them rather. While appropriate when reading an individual text, this rhetorical device limits accessibility when browsing many texts (e.g., on a search engine or on social media). In these scenarios, an explicit conclusion makes for a good candidate summary of an argumentative text. This is especially true if the conclusion is informative, emphasizing specific concepts from the text. With this paper we introduce the task of generating informative conclusions: First, WebisConcluGen-21 is compiled, a large-scale corpus of 136,996 samples of argumentative texts and their conclusions. Second, two paradigms for conclusion generation are investigated; one extractive, the other abstractive in nature. The latter exploits argumentative knowledge that augment the data via control codes and finetuning the BART model on several subsets of the corpus. Third, insights are provided into the suitability of our corpus for the task, the differences between the two generation paradigms, the trade-off between informativeness and conciseness, and the impact of encoding argumentative knowledge. The corpus, code, and the trained models are publicly available. ${ }^{1}$
\end{abstract}

\section{Introduction}

A conclusion of an argument is a statement that conveys a stance towards a specific target (Bar-Haim et al., 2017; Alshomary et al., 2020b). Drawing conclusions is an integral part of argumentation, but often various conclusions may be drawn from a set of premises. Consider the following argumentative text on caffeine adapted from the web: ${ }^{2}$

"Caffeine stimulates the nervous system, signaling fat cells to break down body fat. It also

\footnotetext{
${ }^{1}$ https://github.com/webis-de/ACL-21

${ }^{2}$ https://www.healthline.com/nutrition/top-13-evidence-

based-health-benefits-of-coffee
}

increases epinephrine (adrenaline) levels, a fightor-flight hormone preparing the body for physical exertion. With free body fat acids as fuel, on average, $12 \%$ higher performance is attainable."

Consider further these alternative conclusions:

1. Caffeine is good.

2. Caffeine improves physical performance.

The first conclusion conveys a pro stance towards the target, caffeine. The second, conveys a pro stance towards caffeine, too, but it also emphasizes a specific concept ("physical performance"). The former conclusion is generic, only indicating the stance, while the latter is informative; a distinction also made in text summarization (Section 3$)^{3}$

Argumentative texts include short arguments, such as forum posts and reviews, as well as longform texts, such as essays, blogs, and editorials. Most of these typically have an intended conclusion of which the authors seek to persuade their readers. ${ }^{4}$ While the conclusion may be already implied in a given text, authors often choose not to explicitly provide one, either for rhetorical reasons (Habernal and Gurevych, 2015; Al-Khatib et al., 2016), or to encourage critical thinking (Martin et al., 2003). However, when browsing many argumentative texts (e.g., via a search engine or on a social media timeline), having an explicit conclusion helps human readers (and by extension also machines) to quickly process the texts.

In this paper, we introduce the task of generating informative conclusions for argumentative texts, and take the first steps with four key contributions: (1) Adaptation of the notion of informativeness from text summarization as a desired

\footnotetext{
${ }^{3}$ Other works on argumentation use the term specificity to express a similar idea (Durmus et al., 2019; Ke et al., 2019). ${ }^{4} \mathrm{An}$ exception is an argumentative text dedicated to deliberation, which merely surveys the argument landscape on a given topic without trying to influence the reader's opinion.
} 
property of a conclusion besides stating a target and the stance towards it. (2) Compilation of WebisConcluGen-21, a corpus of 136,996 pairs of argumentative texts and associated conclusions, creating the first large-scale ground truth for conclusion generation. (3) Modeling conclusion generation as an end-to-end task by finetuning a pretrained sequence-to-sequence model, and augmenting the corpus with three types of argumentative knowledge: topic, target, and aspect. (4) Extensive quantitative and qualitative (crowdsourced) evaluation of both the quality of our dataset and the effectiveness of two paradigms for conclusion generation, namely extractive and abstractive approaches.

We present three key findings: (a) Finetuning pretrained language models on our dataset shows strong in-domain performance compared to the extractive approach. (b) Qualitative evaluation shows that the extractive approach generates more informative conclusions, demonstrating a trade-off between conciseness and informativeness. (c) Encoding argumentative knowledge guides the finetuning towards generating argumentative sentences; however, more sophisticated encoding techniques than just using the conventional control codes are needed to generate informative conclusions.

\section{Related Work}

Our work complements and builds on that of A1shomary et al. (2020b), who introduced a conceptual model for conclusion generation, outlining a three-step process: inferring the conclusion's target from the argument's premises, inferring the author's stance towards this target, and generating the conclusion based on these two pieces of information. But Alshomary et al. focused only on the first step of target inference, whereas we model conclusion generation as an end-to-end task.

Conclusion generation can be viewed as a complementary task to summarizing argumentative texts. Previous approaches to the summarization of such texts have been primarily extractive. Egan et al. (2016) proposed summarizing online discussions via "point" extraction, where a point is a verb and its syntactic arguments. Similarly, Bar-Haim et al. (2020) compiled the ArgKP corpus (which we also sample from in Section 4) comprised of arguments for a given topic mapped to key points, composing a summary from a large collection of relevant arguments. Wang and Ling (2016) proposed a data-driven approach using sequence-to-sequence models (Sutskever et al., 2014; Bahdanau et al., 2015) for summarizing movie reviews and debate portal arguments from idebate.org. Several argument mining approaches have also been applied to identify the main claim from arguments (Petasis and Karkaletsis, 2016; Daxenberger et al., 2017). Recently, Alshomary et al. (2020a) proposed a graph-based model using PageRank (Page et al., 1999) that extracts the argument's conclusion and the main supporting reason as an extractive snippet. This model is the core of our extractive summarization approach (Section 5).

A key difference between conclusion generation and general text summarization is the constraint that a conclusion must have a clear stance towards a certain topic. A similar constraint applies to high-quality summaries of long-form argumentative texts such as editorials (Syed et al., 2020), where the persuasiveness of the editorial should be preserved alongside its thesis. Therefore, existing summarization corpora (although large-scale) are unsuitable for studying conclusion generation. A majority of them contain only non-argumentative texts (e.g., news reports) which are more suitable to general-purpose summarization (Kryscinski et al., 2019). Moreover, intrinsic evaluation of summarization corpora has revealed a lower-quality and/or inconsistent ground-truth, rendering them partially unfit for their intended purpose (Bommasani and Cardie, 2020). To fill this gap, we compile WebisConcluGen-21, a large-scale corpus of argumentative texts and their conclusions on diverse topics.

Pre-trained language models have significantly advanced the state-of-the-art in neural text summarization (Liu and Lapata, 2019; Zhang et al., 2019a; Rothe et al., 2020; Huang et al., 2020). However, they have been applied to the domain of argumentation only recently, specifically for argument generation. Gretz et al. (2020) proposed a pipeline based on GPT-2 (Radford et al., 2019) for generating coherent claims for a given debate topic. A more controlled approach for argument generation was developed by Schiller et al. (2020), which performs argument generation with fine-grained control of topic, aspect (core reasoning), and stance. Conclusion generation can be viewed as supplementing argument generation. Ideally, given a conclusion, an argument can be generated constrained by the conclusion's target and stance. To the best of our knowledge, studies investigating pretrained language models for end-to-end conclusion generation 
do not exist. Besides providing a suitable corpus, we analyze the impact of encoding argumentative knowledge in pretrained language models and assess the popular method of control codes (Keskar et al., 2019; Cachola et al., 2020) for encoding the knowledge in our dataset. Furthermore, our qualitative evaluation highlights three key errors (Section 6) arising in the generated outputs that disqualify them as conclusions.

\section{On Informative Conclusions}

In the literature, the conclusion of an argument is the statement that depicts a particular stance towards a certain concept, the target (Walton et al., 2008; Alshomary et al., 2020b). Such a statement is also referred to as the claim of the argument (Toulmin, 2003; Daxenberger et al., 2017). For a longform argumentative text with multiple claims, the conclusion is the main claim that conveys the overall stance towards the subject matter under discussion. The main claim is also known as thesis, or central claim in different genres (Van Dijk, 1995; Burstein and Marcu, 2003; Stab and Gurevych, 2014; Peldszus and Stede, 2015).

The quality of the conclusion of an argumentative text can be assessed in terms of several dimensions, including strength, clarity, and specificity (Ke et al., 2019). Here, a strong connection between argumentation and text summarization can be observed, where the dimension corresponding to specificity is called informativeness. Text summarization distinguishes between indicative and informative summaries. An indicative summary only hints at the principal subject matter of a document to help decide whether to read it (Hovy and Lin, 1998; Kan et al., 2001). An informative summary, on the other hand, covers the main information in the source document, ideally serving as its surrogate (Maybury, 1999).

The conceptual connection between argumentation and summarization could be described as follows: the informativeness of a conclusion is closely connected to the specificity dimension, in the sense that an informative conclusion must be specific to allow for a better understanding of an argumentative text's gist. Seeing that "specificity" and "informativeness" may be used interchangeably, we opted for the latter and the term "informative conclusion" here, to underline the connection.

In contrast to indicative conclusions, which broadly convey (implicitly or explicitly) the stance towards a topic (e.g., "Caffeine is good."), informative conclusions also discuss specific concepts from (or implied by) the argumentative text (e.g., "Caffeine improves physical performance.”). Concepts of the argumentative text exemplified in Section 1 may refer to the topic (e.g., "Is coffee beneficial?"), the target of the conclusion (e.g., "caffeine"), or a specific aspect (e.g.,"energy levels").

\section{The Webis-ConcluGen-21 Corpus}

This section details the construction of the Webis Conclusion Generation Corpus 2021 (WebisConcluGen-21), a corpus of 136,996 pairs of argumentative texts and conclusions covering diverse topics. The corpus is derived from two reliable sources, where the conclusions of argumentative texts are explicitly identifiable: Reddit's ChangeMyView forum and debate corpora.

\subsection{Data Source: Reddit's ChangeMyView}

ChangeMyView (CMV) is an online forum for persuasive discussions that start with a user who presents a view and asks others to challenge it. The forum's rules strictly enforce that (1) users' posts must contain sufficient reasoning, (2) posts must take a stance (and not be neutral), and (3) the title of a post must sufficiently sum up an author's view (as a statement and not a question). ${ }^{5}$ Given these constraints, the original post of a discussion can be operationalized as an argumentative text, and the corresponding title as its (intended) conclusion. Starting from the Reddit crawls provided by Baumgartner et al. (2020), we compiled 61,695 such pairs by processing all CMV discussions up until August 2019. The included posts are those whose argumentative text was longer than ten words, the conclusion longer than two words, and the title includes the "CMV" tag. ${ }^{6}$ An average argumentative text is 312 words long and a conclusion 15 words.

To better understand the relation of the conclusions to their respective argumentative texts, and the expected difficulty of generating them, we analyzed a sample of 200 pairs manually. ${ }^{7}$ Table 1

\footnotetext{
${ }_{5}$ https://reddit.com/r/changemyview/wiki/rules

${ }^{6}$ These heuristics reflect manual inspections, and the fact that we did not wish to compile a representative sample of ChangeMyView's discussions, but a purposeful selection of highquality pairs of argumentative texts and their conclusions: In light of this, the lower bounds are still quite inclusive with respect to extremely short samples.

${ }^{7}$ These examples were taken from the Dec-2019 Reddit submissions to ensure a truly-hidden sample as BART was originally trained on the OpenWebText dataset containing samples
} 


\begin{tabular}{llr}
\hline Type & Description & $\%$ \\
\hline Extractive & $\begin{array}{l}\text { Conclusion is present verbatim in the } \\
\text { argumentative text. }\end{array}$ & 12.8 \\
Paraphrase & $\begin{array}{l}\text { Conclusion is synonymous to, or a fu- } \\
\text { sion of a part of the argumentative text. }\end{array}$ & 24.1 \\
Abstractive & $\begin{array}{l}\text { Conclusion is inferred from the argu- } \\
\text { mentative text. }\end{array}$ & 57.8 \\
\hline No conclusion & $\begin{array}{l}\text { Conclusion cannot be derived from the } \\
\text { argumentative text. }\end{array}$ & 5.3 \\
\hline
\end{tabular}

Table 1: Different types of conclusions in $200 \mathrm{CMV}$ samples, and their relative proportion.

shows the proportion of extractive, paraphrased, and abstractive conclusions in our sample, where the former only need to be extracted, and the latter demand actual text synthesis. Paraphrases share aspects of both, though arguably, extracting the paraphrased part would suffice. Altogether, CMV provides for $94.7 \%$ valid pairs of argumentative texts and conclusions at sufficiently low noise $(5.3 \%)$. The amount of non-trivial conclusions (abstractive + paraphrase) are sufficiently challenging, as found in our qualitative evaluation (Section 6).

\subsection{Data Source: Debate Corpora}

Online debate portals facilitate semi-structured debates on controversial topics, where pro and con arguments or argumentative texts are collected. Conclusions are clearly stated even for individual arguments. Given their high-quality curation, debate portals constitute the majority of argument corpora. We utilized the following existing corpora:

Kialo is a debate platform that enables "visual reasoning" in complex debates via a tree-based structure (Chaudoin et al., 2017). A key advantage here is the role of moderators in curating accepted arguments, rendering it a rich resource (Durmus et al., 2019). As debates progress, the arguments are reorganized into multiple hierarchies, each with a conclusion at its root. ${ }^{8}$ We compiled this corpus from scratch in accordance with the website's terms and conditions. In 1,640 English discussions, at each level of the discussion tree, all pro arguments were matched to the corresponding root conclusion, obtaining a total of 82,728 examples.

Args.me is a search engine (Wachsmuth et al., 2017) indexing the Args.me Corpus (Ajjour et al., 2019b), comprised of argumentative texts, their

from Reddit (Liu et al., 2019; Radford et al., 2019).

${ }^{8}$ For an example, see: https://www.kialo.com/pro-life-vs-prochoice-should-abortion-be-legal-5637 conclusions and their stance from four debate portals: debatewise.org, idebate.org, debatepedia.org, and debate.org. We used the "cleaned" version of this corpus containing 387,606 samples and applied further post-processing. On manual inspection, we observed that a number of examples from debate.org contained spam, sarcasm, or ad hominem attacks, or they were not self-contained due to references to previous turns. To avoid noise, we excluded all examples from this portal. Next, we removed arguments with con stance towards a conclusion. ${ }^{9}$ This is due to the fact that considering these examples for training would first require negating their conclusions to reflect the con stance. We leave such automatic claim negation (Bilu et al., 2015) for future work. Finally, to favor informative conclusions, we excluded arguments whose conclusion was the same as the discussion topic (which is generally indicative). This heavy filtering resulted in a total of 23,448 argument-conclusion pairs.

ArgsKP is a corpus of arguments and a set of key points written by domain experts on 28 topics (Bar-Haim et al., 2020). For each topic, the corpus contains multiple arguments which have been mapped via crowdsourcing to their respective key points. From this corpus, we obtained 2,341 pairs; again, only pro arguments and those that have been mapped to a specific key point, the conclusion.

Postprocessing. The structure of debate portals allows for multiple arguments to be mapped to a single conclusion. This happens when different users independently contribute pro and con arguments, which is acceptable, since the same conclusion can be drawn from different arguments with different frames (Ajjour et al., 2019a). Apart from the ones filtered in preprocessing the debates corpora, we preserved duplicate conclusions across debates as their arguments are still unique. Similar to CMV, the included argumentative texts were those whose length exceeded ten words. Also, argumentative texts shorter than their conclusion were excluded. This removed many pairs from the Kialo discussions. Altogether, we retained 75,301 usable examples from all three corpora.

\subsection{Corpus Statistics}

The argumentative texts are on average longer in CMV (312 words) compared to those in debates (44.5 words). A reason is that, on debate portals, each argumentative text seems to be a selfcontained argument. CMV posts, by comparison,

${ }^{9}$ This does not exclude conclusions that are already negations. 
often contain multiple arguments and/or preface the actual argument with additional background. However, the corresponding conclusions are of similar length (15 words for CMV and 18.4 words for debates on average, about the length of an average English sentence). For both data sources, we measured the percentage of words in a conclusion that do not occur in the argumentative text as a measure of "novelty" (Narayan et al., 2018). For CMV, the average novelty is $33.2 \%$, and for debates, the novelty is $81.6 \%$, which is due to the fact that multiple arguments have been mapped to a single conclusion, and that arguments supporting (or attacking) a conclusion during an ongoing discussion are usually not directly derived from it.

\section{Generating Informative Conclusions}

Given the mixture of conclusion types shown in Table 1, we approach the generation of informative conclusions according to two paradigms, one extractive approach combined with paraphrasing, and one abstractive approach combined with state-ofthe-art argument mining technology.

\subsection{Paraphrased Conclusion Generation}

Paraphrased conclusions are fundamentally extractive in nature, where an extracted sentence is reformulated to improve it. To extract conclusions, we employ the graph-based approach of Alshomary et al. (2020a), originally designed to generate snippets for argument search results. Given an argument, a snippet is generated as follows: (1) related arguments are retrieved as context, (2) all argument's sentences and those from the retrieved ones are embedded, (3) the PageRank of the sentences is computed, and lastly (4) the argument's two topranked sentences are returned. Underlying this approach is the hypothesis that an extractive snippet for an argument should comprise its conclusion and its most important supporting premise. Sentences are thus scored regarding their centrality in context of other arguments and their argumentativeness.

Our goal is to generate a single conclusion statement, thus we consider only the top-ranked sentence as the conclusion from the approach of Alshomary et al. (2020a). This sentence is automatically paraphrased using PEGASUS (Zhang et al., 2020a), finetuned on the Google PAWS dataset (Zhang et al., 2019b). ${ }^{10}$ For instance, consider the

\footnotetext{
${ }^{10} \mathrm{https} / / /$ huggingface.co/tuner007/pegasus_paraphrase
}

top-ranked sentence from a post questioning the use of hormone blockers on transgender kids: ${ }^{11}$

"I don't see it as anything different, and I think it is scandalous to permanently change a child's entire life on a whim rather than treating their mental health."

After paraphrasing, it reads as follows:

"I think it's scandalous to change a child's life on a whim, rather than treating their mental health, and I don't see it as anything different."

The paraphraser primarily rearranges the sentence; and shared phrases with the original are typical in the paraphrased sentences we reviewed. This approach, called Arg-PageRank, represents an advanced extractive paradigm.

\subsection{Abstractive Conclusion Generation}

Abstractive conclusions can be formulated freely, provided they capture the main pieces of information required for an informative conclusion: topic, targets, stance, and aspects. In this regard, our approach is three-fold (see Figure 1): (1) Automatic extraction of the aforementioned pieces of information from a given argumentative text; (2) augmentation of the training examples in Webis-ConcluGen21 using control codes, and (3) domain transfer of a pretrained abstractive news summarization model via finetuning on the augmented corpus.

Argumentative Knowledge Extraction. This step details our respective approaches at providing the prerequisite pieces of information to formulate an informative conclusion, namely topic, targets, and aspects. Table 2 shows an example.

Topic: An argumentative text's topic is a description of what it is about. For argumentative texts from debates, we use the associated debate title as the topic. For CMV posts, their titles are also their conclusions; here, topic information is considered missing (denoted as 'NA' token).

Targets: The target of a conclusion is typically a controversial concept or statement (Bar-Haim et al., 2017). For an argumentative text, though, an overlap with its topic is possible, different targets can also be found in its premises. Moreover, when not explicitly stated, the targets of a conclusion can be inferred from either the targets of premises, or external knowledge bases. A set of possible targets

\footnotetext{
${ }^{11} \mathrm{https} / / / \mathrm{www} . \mathrm{re} d$ dit.com/r/changemyview/comments/ e97sir/cmv_giving_children_puberty_blockers_to_allow/
} 


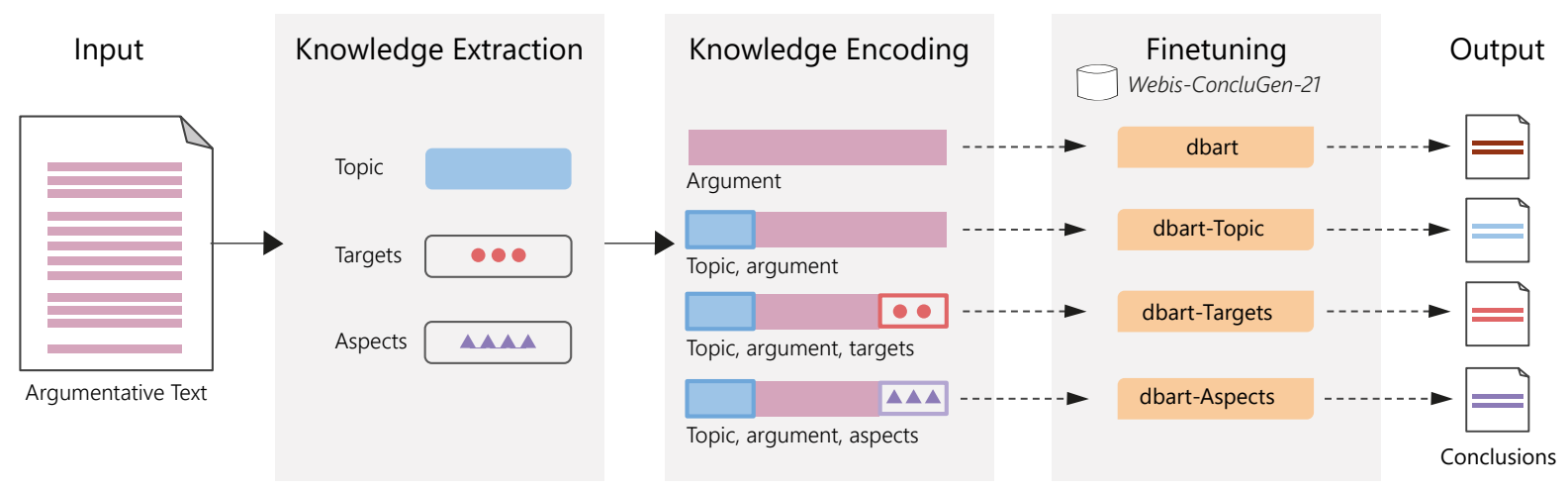

Figure 1: The three steps of our approach to abstractive conclusion generation: For all examples in the WebisConcluGen-21 corpus (1) different pieces of argument knowledge are extracted namely the discussion topic, possible conclusion targets, and covered aspects, (2) this knowledge is encoded using control codes, and (3) knowledgespecific variations are finetuned of the distilled BART model to generate informative conclusions.

\begin{tabular}{|c|c|}
\hline Argument & $\begin{array}{l}\text { Feminism as a 'linguistic term' often misses clarity, universal definition and regularly incorporates opposite } \\
\text { goals at the same time in regard to key feminist issues as gender equality, gender-neutrality, non-binary and } \\
\text { gender-related rights. The linguistic term thereby clouds public debate and hampers the setting of clear } \\
\text { social and political goals in society. }\end{array}$ \\
\hline Conclusion & $\begin{array}{l}\text { Feminism is an umbrella of ideologies first and foremost, and consequently, it muddies the discussion of } \\
\text { gender equality with its ideological baggage. }\end{array}$ \\
\hline Topic & Is Feminism a Force For Good? \\
\hline Aspects & $\begin{array}{l}\text { clouds, gender equality, non-binary, opposite goals, public debate, gender-related rights, clarity, gender- } \\
\text { neutrality, social and political goals, universal definition }\end{array}$ \\
\hline Targets & The linguistic term, Feminism as a 'linguistic term' \\
\hline $\begin{array}{l}\text { Encoded } \\
\text { Representation }\end{array}$ & $\begin{array}{l}<|T O P I C|>\text { Is Feminism a Force For Good? }<|A R G U M E N T|>\text { Feminism as a 'linguistic term' often misses } \\
\text { clarity, universal definition and regularly incorporates opposite goals at the same time in regard to key } \\
\text { feminist issues as gender equality, gender-neutrality, non-binary and gender-related rights. The linguistic } \\
\text { term thereby clouds public debate and hampers the setting of clear social and political goals in soci- } \\
\text { ety. }<|T A R G E T S|>\text { The linguistic term, Feminism as a 'linguistic term }<|C O N C L U S I O N|>\end{array}$ \\
\hline
\end{tabular}

Table 2: Example argument-conclusion pair along with topic, targets, and aspects. The last row shows the representation for finetuning models on specific types of encoded external knowledge (here, on conclusion targets).

for every argumentative text in the corpus are automatically identified using the target identification model of Alshomary et al. (2020b).

Aspects: Text spans that contribute to the core reasoning of an argument are called its aspects (Schiller et al., 2020). Aspects can be viewed as subtopics related to the main topic of an argumentative text, encoding a stance. Including aspects into a conclusion can render it more specific and, thus, informative. We identify aspects for all samples in the corpus, using the model of Schiller et al. This model trains a BERT-based (Devlin et al., 2019) ranker on a corpus containing 5,032 high-quality argumentative sentences that are manually labeled with aspects at the token level.

Stance is excluded as an explicit input to our models. For CMV, by design, a post supports its title. For debate portals, only argumentative texts with pro stance towards their conclusion have been considered. Nevertheless, argumentative texts and their conclusions in our corpus may, implicitly or explicitly, express their own stance towards implicit or explicit targets. Implicit stance can be encoded via the aspects.

Argumentative Knowledge Encoding. The extracted pieces of knowledge are encoded into a training example with control codes using special tokens (Cachola et al., 2020): <|TOPIC| $\rangle,\langle| A R$ GUMENT $|>,<| A S P E C T S|>,<| T A R G E T S \mid>$, and $<|C O N C L U S I O N|>$. Table 2 shows a corresponding example input sequence encoding the topic and the conclusion targets. To examine the impact of individual knowledge types, we create three versions of Webis-ConcluGen-21: topic-encoded, aspectencoded, and target-encoded. Presuming the availability of a topic in nearly all real-world applications, it is also encoded in the latter two versions. Since aspects and targets overlap in $38.3 \%$ of the case in the corpus, they are independently encoded. 


\begin{tabular}{llrr}
\hline Model & Data & \#Train & \#Valid \\
\hline dbart-XSum & XSum & 204,045 & $\mathrm{n} / \mathrm{a}$ \\
dbart-CMV & CMV & 55,768 & 5,577 \\
dbart-Debates & Debates & 67,770 & 6,777 \\
dbart & All & 123,538 & 12,354 \\
dbart-Topic & All+topic & 123,538 & 12,354 \\
dbart-Aspects & All+topic+aspects & 122,040 & 12,192 \\
dbart-Targets & All+topic+targets & 110,867 & 11,068 \\
Arg-PageRank & none, unsupervised model & \\
\hline
\end{tabular}

Table 3: Corpus splits for all six variants. 'All' refers to the entire Webis-ConcluGen-21 corpus. Models were automatically evaluated on a test set of 1,000 examples, and qualitatively on 300 examples (Section 6).

\begin{tabular}{lc}
\hline Parameter & Value \\
\hline max_target_length & 100 \\
warmup_steps & 500 \\
eval_steps & 500 \\
attention_dropout & 0.1 \\
label_smoothing & 0.1 \\
sampling & sortish_sampler \\
seed & 5153 \\
num_beams & 6 \\
length_penalty & 0.5 \\
gradient_accumulation_steps & 1 \\
lr_scheduler & linear \\
\hline
\end{tabular}

Table 4: Hyperparameters for finetuning BART.

Finetuning. As conclusion generation is closely related to abstractive text summarization, we picked BART (Lewis et al., 2020), a pretrained state-ofthe-art summarization model, for finetuning on the three augmented versions of Webis-ConcluGen21 . However, BART has approximately $10 \%$ more parameters than BERT, which makes it resourceintensive for finetuning. To account for this, we used the distilled checkpoint derived using the "shrink-and-finetune" approach of Shleifer and Rush (2020), where large sequence-to-sequence models are compressed by extracting "distilled student models" (Sanh et al., 2019) from a teacher model (here, BART). We used distilled BART finetuned on the XSum corpus (Narayan et al., 2018) (dbart-XSum) provided by the Transformers library (Wolf et al., 2020), ${ }^{12}$ since the average length of our ground-truth conclusions is similar to the summaries in XSum. Additionally, we also added our control codes as special tokens to the BART tokenizer during finetuning in order to avoid splitting them into sub-word tokens while processing the encoded sequences.

We first applied dbart-XSum on the held-out test set of 200 examples analyzed for Table 1 to evaluate the domain transfer from news reports to argumentative texts. On manual evaluation, $79.1 \%$ of

\footnotetext{
${ }^{12} \mathrm{https}: / /$ huggingface.co/sshleifer/distilbart-xsum-12-6
}

\begin{tabular}{lrrrr}
\hline Model & \multicolumn{1}{c}{ BERTScore (F1) } & Rou.-1 & Rou.-2 & Rou.-L \\
\hline dbart-XSum & 0.21 & 15.28 & 3.10 & 13.31 \\
dbart-CMV & 0.32 & 20.35 & 7.11 & 18.80 \\
dbart-Debates & 0.23 & 15.38 & 4.85 & 14.22 \\
dbart & $\mathbf{0 . 3 9}$ & $\mathbf{3 1 . 7 3}$ & $\mathbf{1 9 . 4 8}$ & $\mathbf{3 0 . 8 7}$ \\
dbart-Topic & 0.34 & 23.74 & 9.56 & 22.14 \\
dbart-Aspects & 0.33 & 23.47 & 9.46 & 22.01 \\
dbart-Targets & 0.34 & 23.80 & 9.63 & 22.25 \\
Arg-PageRank & 0.20 & 15.35 & 3.20 & 13.37 \\
\hline
\end{tabular}

Table 5: Automatic evaluation of models on the internal test set consisting of 1,000 pairs (500 each from CMV and Debates). BERTScore is the re-scaled F1 score; in addition, average Rouge-1, -2 , and -L are reported.

the outputs were invalid conclusions, primarily due to being non-argumentative (Section 6). This demonstrates that existing summarization models are ineffective when applied on argumentative texts and must be trained on task-specific data.

\subsection{Training Details}

We compiled six variations of the corpus (with and without encoded knowledge) for finetuning the The dbart-XSum model with 306M parameters. ${ }^{12}$ Table 3 shows the training and validation splits for each model variant and the corresponding data subsets, and Table 4 shows the chosen hyperparameters. The standard finetuning regimen was employed from the Transformers library ${ }^{13}$ to train each model on a V100 GPU for 6 epochs with batch size 1 , dropout rate 0.1 , adafactor optimizer, learning rate of $3 e-5$, and beam search for inference. For dbart $-<\mathrm{CMV} \mid$ Debates $\mid \mathrm{Al} 1>$ the maximum source sequence length was set to 512 tokens, while for dbart-<Topic|Aspects|Targets $>$ we increased it to 750 tokens to account for the appended knowledge in the input sequence. On a single V100 GPU, the runtime varies between 3 to 5 days per model, depending on their corresponding training splits.

\section{Evaluation}

Our models are evaluated via both: (1) An automatic evaluation on a large test set using standard metrics, and (2) a manual evaluation on a smaller test set via crowdsourcing.

\subsection{Automatic Evaluation}

On a test set of 1,000 examples with known groundtruth (500 each from CMV and from the debate corpora), we computed ROUGE (Lin, 2004) ${ }^{14}$ and BERTScore (Zhang et al., 2020b) ${ }^{15}$ for all models.

\footnotetext{
${ }^{13} \mathrm{https} / / /$ github.com/huggingface/transformers/tree/master/ examples/legacy/seq2seq

${ }^{14}$ https://github.com/pltrdy/rouge

${ }^{15}$ https://github.com/Tiiiger/bert_score
} 
Table 5 shows that dbart-Xsum performs poorly on argumentative texts. Inspecting the reasons for this shortcoming, we found several outputs of the model to be either neutral sentences (despite having the right target), or hallucinations with artifacts from the XSum corpus (e.g., "In our series of letters from African journalists [...]" or "This week I've been writing about [...]"). Among the finetuned models, dbart, trained on the entire corpus without any encoded knowledge, performs best across all metrics. The knowledge-encoded models exert a drop in effectiveness, but still outperform models trained on the sub-datasets dbart-CMV and dbart-Debates.

All finetuned models generate concise outputs of similar lengths (average 12 words), while Arg-PageRank extracts longer spans (25 words). Outputs of the knowledge-encoded models are somewhat similar to each other (average pairwise Jaccard similarity of 0.43), compared to those from dbart ( 0.27 with any knowledge-encoded model).

\subsection{Manual Evaluation}

Given the results of the automatic evaluation, only the models trained on the entire corpus were manually evaluated against our baseline approach Arg-PageRank. A test set of 300 examples was employed, 100 each from debates and CMV posts, plus 100 comments to CMV posts. The latter include only comments with at least 100 words and exclude non-argumentative ones as per automatic claim-detection (Chakrabarty et al., 2019). This part of the test set corresponds to an unsupervised evaluation of the conclusions, since no ground truth for the comments is available.

Two expert writers, both native English speakers, were hired via Upwork.com. ${ }^{16}$ For every given argumentative text in the test set, all candidate conclusions generated by the different models were shown to the annotators in random order, and without revealing the respective model's name. Assessment was cast as a series of binary decisions: first, whether a given candidate is a conclusion, and if yes, whether it is fluent, and whether it is informative. To simplify judging informativeness, we only asked if the conclusion was too generic. For each candidate judged not to be a conclusion, we asked whether it either has the (1) wrong target (WT), conveys the (2) wrong stance (WS), or whether it is (3) non-argumentative (NA).

Table 6 shows the percentage of cases on which both annotators agreed. For CMV and debates,

\footnotetext{
${ }^{16}$ An hourly rate of about 30 USD was paid.
}

\begin{tabular}{lrrrrr}
\hline Model & Concl. & Inform. & \multicolumn{3}{c}{ Error Types } \\
\cline { 4 - 6 } & & & WT & WS & NA \\
\hline CMV Posts & & & & & \\
dbart & $36 \%$ & $4 \%$ & $56 \%$ & $22 \%$ & $22 \%$ \\
dbart-Topic & $28 \%$ & $0 \%$ & $59 \%$ & $23 \%$ & $18 \%$ \\
dbart-Aspects & $33 \%$ & $6 \%$ & $69 \%$ & $23 \%$ & $8 \%$ \\
dbart-Targets & $27 \%$ & $4 \%$ & $69 \%$ & $23 \%$ & $8 \%$ \\
Arg-PageRank & $11 \%$ & $7 \%$ & $0 \%$ & $0 \%$ & $100 \%$ \\
\hline Debates & & & & & \\
dbart & $14 \%$ & $6 \%$ & $65 \%$ & $9 \%$ & $26 \%$ \\
dbart-Topic & $14 \%$ & $3 \%$ & $76 \%$ & $12 \%$ & $12 \%$ \\
dbart-Aspects & $7 \%$ & $2 \%$ & $77 \%$ & $13 \%$ & $10 \%$ \\
dbart-Targets & $11 \%$ & $2 \%$ & $71 \%$ & $17 \%$ & $12 \%$ \\
Arg-PageRank & $10 \%$ & $6 \%$ & $7 \%$ & $0 \%$ & $93 \%$ \\
\hline Comments & & & & & \\
dbart & $12 \%$ & $2 \%$ & $52 \%$ & $18 \%$ & $30 \%$ \\
dbart-Topic & $6 \%$ & $2 \%$ & $58 \%$ & $24 \%$ & $18 \%$ \\
dbart-Aspects & $7 \%$ & $3 \%$ & $52 \%$ & $33 \%$ & $15 \%$ \\
dbart-Targets & $8 \%$ & $3 \%$ & $55 \%$ & $35 \%$ & $10 \%$ \\
Arg-PageRank & $17 \%$ & $9 \%$ & $5 \%$ & $5 \%$ & $90 \%$ \\
\hline
\end{tabular}

Table 6: Full agreement percentages of two annotators on 300 examples, grouped by the example type (posts, debates, comments). The first column is the $\%$ of valid conclusions, the second the $\%$ of informative conclusions, followed by the \% distribution of error types (lower is better) of a model. On average, all models were judged to be fluent for $97 \%$ of the conclusions.

finetuning outperforms Arg-PageRank at generating conclusions that convince the experts: dbart performs best on CMV (36\%), and dbart and dbart-Topic on debates (14\%).

Comments appear to be a particularly difficult type of test cases. This is because comments to the first post may not be self-contained but refer back to the post, they may have a mixed stance (supporting only part of the post while opposing the rest), and they may introduce new targets and aspects (different concepts)—based on our inspection of the comments. In such cases, extracting the conclusion from the comment (and paraphrasing it) using Arg-PageRank performs best (17\%).

Encoding knowledge slightly impacts the effectiveness. Across all example types, knowledgeencoded models perform equally well, sometimes worse, sometimes better than dbart. Encoding topic with aspects or targets performs better on posts and comments.

As for informativeness, dbart-Aspects generates a higher number of informative conclusions for posts, while dbart does best in debates, among the finetuned models. In all domains, Arg-PageRank performs similar to or better than all approaches due to extracting claims that are twice as long on average (24 words) compared to the finetuned models (12 words), hence capturing more information. 
Inspecting the error types, encoding argumentative knowledge increases the number of argumentative candidate conclusions, validating its positive impact. All knowledge-encoded models have fewer non-argumentative (NA) errors compared to dbart. However, this affects target inference; the knowledge-encoded models generate more wrong targets (WT). The mixed stance of comments (supporting part of the original post, while opposing the rest) leads to a higher number of stance errors (WS) for dbart-Aspects and dbart-Targets. Finally, for Arg-PageRank, almost all errors were nonargumentative sentences (NA).

\subsection{Discussion}

Our qualitative evaluation indicates that generating informative conclusions is challenging, and that our data is well-suited for the task, due to a mix of conclusion types (Table 1), and diverse data sources. Leveraging external knowledge, though a promising feature for guiding finetuning, may benefit from better encoding strategies compared to the conventional method of using control codes in text. However, given that the identified knowledge is extractive and that we encoded multiple aspects and targets per example in contrast to related controlled text generation approaches (Keskar et al., 2019; Schiller et al., 2020; Gretz et al., 2020; Cachola et al., 2020), further investigations with importance sampling of argumentative knowledge are advised. Ideally, such sampling would be tailored to a specific domain or target audience.

Likewise, regarding the informativeness of the generated conclusions, a trade-off between conciseness and specificity must be decided. Our experiments suggest that long extractive conclusions capture more information compared to the more concise (and fluent) abstractive one of the finetuned models, rendering them preferable to the annotators when sufficient background is missing. Finally, for comments, modeling the argumentative context supplemented by explicit stance identification is necessary to generate valid conclusions.

\section{Conclusion}

The notion of an informative conclusion is introduced and discussed in the context of computational argumentation as well as text summarization. Informative conclusions are to argumentation what brief summaries are to text: they concisely convey its main points. We lay the foundation for studying the conclusions of argumentative texts, compiling the Webis-ConcluGen-21 corpus, comprising 136,996 pairs of argumentative texts and corresponding conclusions.

Conclusions are diverse and typically depart significantly from the argumentative text they are derived from, paraphrasing it, and more than half the time abstracting over it. Authors typically tailor their conclusions to the occasion; and in many cases, they are not necessarily made explicit. This is where we contribute by tackling the task of generating an informative conclusion. The two main paradigms we study — paraphrased (incl. extractive) vs. abstractive conclusion generation-compete closely with each other.

\section{Ethics Statement}

Our dataset is a collection of opinionated texts obtained from sources that are available publicly and acknowledged appropriately. We respected their terms and conditions.

We did not employ any author-specific features in our approaches and instead processed only the corresponding arguments, although representing personal views of anonymous authors.

The proposed technology will be applicable to an English-speaking audience. While failures in generating valid conclusions may mislead a reader's initial interpretation of an argument, we do not aim at applications that prevent readers from reading the complete arguments. Rather, we seek to simplify the consumption of public discussions comprising several arguments by providing explicit, informative conclusions especially for longer arguments.

Finally, in terms of computational resources, we restricted ourselves to the smaller, distilled checkpoints of a large pretrained model that can be trained with (comparably) smaller resources and are accessible to majority of the researchers.

\section{Acknowledgments}

We thank the reviewers for their valuable feedback. This work was supported by the German Federal Ministry of Education and Research (BMBF, 01/S18026A-F) by funding the competence center for Big Data and AI (ScaDS.AI Dresden/Leipzig). Computations for this work were done (in part) using resources of the Leipzig University Computing Centre, who we sincerely thank for their support. 


\section{References}

Yamen Ajjour, Milad Alshomary, Henning Wachsmuth, and Benno Stein. 2019a. Modeling frames in argumentation. In Proceedings of the 2019 Conference on Empirical Methods in Natural Language Processing and the 9th International Joint Conference on Natural Language Processing (EMNLPIJCNLP), pages 2922-2932, Hong Kong, China. Association for Computational Linguistics.

Yamen Ajjour, Henning Wachsmuth, Johannes Kiesel, Martin Potthast, Matthias Hagen, and Benno Stein. 2019b. Data acquisition for argument search: The args.me corpus. In KI 2019: Advances in Artificial Intelligence - 42nd German Conference on AI, Kassel, Germany, September 23-26, 2019, Proceedings, pages 48-59.

Khalid Al-Khatib, Henning Wachsmuth, Johannes Kiesel, Matthias Hagen, and Benno Stein. 2016. A news editorial corpus for mining argumentation strategies. In Proceedings of COLING 2016, the 26th International Conference on Computational Linguistics: Technical Papers, pages 3433-3443, Osaka, Japan. The COLING 2016 Organizing Committee.

Milad Alshomary, Nick Düsterhus, and Henning Wachsmuth. 2020a. Extractive snippet generation for arguments. In Proceedings of the 43rd International ACM SIGIR conference on research and development in Information Retrieval, SIGIR 2020, Virtual Event, China, July 25-30, 2020, pages 19691972. ACM.

Milad Alshomary, Shahbaz Syed, Martin Potthast, and Henning Wachsmuth. 2020b. Target inference in argument conclusion generation. In Proceedings of the 58th Annual Meeting of the Association for Computational Linguistics, pages 4334-4345, Online. Association for Computational Linguistics.

Dzmitry Bahdanau, Kyunghyun Cho, and Yoshua Bengio. 2015. Neural machine translation by jointly learning to align and translate. In 3rd International Conference on Learning Representations, ICLR 2015, San Diego, CA, USA, May 7-9, 2015, Conference Track Proceedings.

Roy Bar-Haim, Indrajit Bhattacharya, Francesco Dinuzzo, Amrita Saha, and Noam Slonim. 2017. Stance classification of context-dependent claims. In Proceedings of the 15th Conference of the European Chapter of the Association for Computational Linguistics: Volume 1, Long Papers, pages 251-261, Valencia, Spain. Association for Computational Linguistics.

Roy Bar-Haim, Lilach Eden, Roni Friedman, Yoav Kantor, Dan Lahav, and Noam Slonim. 2020. From arguments to key points: Towards automatic argument summarization. In Proceedings of the 58th Annual Meeting of the Association for Computational Linguistics, ACL 2020, Online, July 5-10, 2020, pages 4029-4039. Association for Computational Linguistics.

Jason Baumgartner, Savvas Zannettou, Brian Keegan, Megan Squire, and Jeremy Blackburn. 2020. The pushshift reddit dataset. In Proceedings of the Fourteenth International AAAI Conference on Web and Social Media, ICWSM 2020, Held Virtually, Original Venue: Atlanta, Georgia, USA, June 8-11, 2020, pages 830-839. AAAI Press.

Yonatan Bilu, Daniel Hershcovich, and Noam Slonim. 2015. Automatic claim negation: Why, how and when. In Proceedings of the 2nd Workshop on Argumentation Mining, pages 84-93, Denver, CO. Association for Computational Linguistics.

Rishi Bommasani and Claire Cardie. 2020. Intrinsic evaluation of summarization datasets. In Proceedings of the 2020 Conference on Empirical Methods in Natural Language Processing (EMNLP), pages 8075-8096, Online. Association for Computational Linguistics.

Jill Burstein and Daniel Marcu. 2003. A machine learning approach for identification of thesis and conclusion statements in student essays. Computers and the Humanities, 37(4):455-467.

Isabel Cachola, Kyle Lo, Arman Cohan, and Daniel S. Weld. 2020. TLDR: extreme summarization of scientific documents. In Proceedings of the 2020 Conference on Empirical Methods in Natural Language Processing: Findings, EMNLP 2020, Online Event, 16-20 November 2020, pages 4766-4777. Association for Computational Linguistics.

Tuhin Chakrabarty, Christopher Hidey, and Kathy McKeown. 2019. IMHO fine-tuning improves claim detection. In Proceedings of the 2019 Conference of the North American Chapter of the Association for Computational Linguistics: Human Language Technologies, Volume 1 (Long and Short Papers), pages 558-563, Minneapolis, Minnesota. Association for Computational Linguistics.

Stephen Chaudoin, J Shapiro, and Dustin Tingley. 2017. Revolutionizing teaching and research with a structured debate platform1. Journal of Political Science, 58:1064-1082.

Johannes Daxenberger, Steffen Eger, Ivan Habernal, Christian Stab, and Iryna Gurevych. 2017. What is the essence of a claim? cross-domain claim identification. In Proceedings of the 2017 Conference on Empirical Methods in Natural Language Processing, pages 2055-2066, Copenhagen, Denmark. Association for Computational Linguistics.

Jacob Devlin, Ming-Wei Chang, Kenton Lee, and Kristina Toutanova. 2019. BERT: Pre-training of deep bidirectional transformers for language understanding. In Proceedings of the 2019 Conference of the North American Chapter of the Association for Computational Linguistics: Human Language 
Technologies, Volume 1 (Long and Short Papers), pages 4171-4186, Minneapolis, Minnesota. Association for Computational Linguistics.

Esin Durmus, Faisal Ladhak, and Claire Cardie. 2019. Determining relative argument specificity and stance for complex argumentative structures. In Proceedings of the 57th Annual Meeting of the Association for Computational Linguistics, pages 4630-4641, Florence, Italy. Association for Computational Linguistics.

Charlie Egan, Advaith Siddharthan, and Adam Wyner 2016. Summarising the points made in online political debates. In Proceedings of the Third Workshop on Argument Mining (ArgMining2016), pages 134-143, Berlin, Germany. Association for Computational Linguistics.

Shai Gretz, Yonatan Bilu, Edo Cohen-Karlik, and Noam Slonim. 2020. The workweek is the best time to start a family - A study of GPT-2 based claim generation. In Proceedings of the 2020 Conference on Empirical Methods in Natural Language Processing: Findings, EMNLP 2020, Online Event, 16-20 November 2020, pages 528-544. Association for Computational Linguistics.

Ivan Habernal and Iryna Gurevych. 2015. Exploiting debate portals for semi-supervised argumentation mining in user-generated web discourse. In Proceedings of the 2015 Conference on Empirical Methods in Natural Language Processing, pages 21272137, Lisbon, Portugal. Association for Computational Linguistics.

Eduard Hovy and Chin-Yew Lin. 1998. Automated text summarization and the Summarist system. In TIPSTER TEXT PROGRAM PHASE III: Proceedings of a Workshop held at Baltimore, Maryland, October 13-15, 1998, pages 197-214, Baltimore, Maryland, USA. Association for Computational Linguistics.

Dandan Huang, Leyang Cui, Sen Yang, Guangsheng Bao, Kun Wang, Jun Xie, and Yue Zhang. 2020 What have we achieved on text summarization? In Proceedings of the 2020 Conference on Empirical Methods in Natural Language Processing (EMNLP), pages 446-469, Online. Association for Computational Linguistics.

Min-Yen Kan, Kathleen R. McKeown, and Judith L. Klavans. 2001. Applying natural language generation to indicative summarization. In Proceedings of the ACL 2001 Eighth European Workshop on Natural Language Generation (EWNLG).

Zixuan Ke, Hrishikesh Inamdar, Hui Lin, and Vincent Ng. 2019. Give me more feedback II: Annotating thesis strength and related attributes in student essays. In Proceedings of the 57th Annual Meeting of the Association for Computational Linguistics, pages 3994-4004, Florence, Italy. Association for Computational Linguistics.
Nitish Shirish Keskar, Bryan McCann, Lav R. Varshney, Caiming Xiong, and Richard Socher. 2019. CTRL: A conditional transformer language model for controllable generation. CoRR, abs/1909.05858.

Wojciech Kryscinski, Nitish Shirish Keskar, Bryan McCann, Caiming Xiong, and Richard Socher. 2019. Neural text summarization: A critical evaluation. In Proceedings of the 2019 Conference on Empirical Methods in Natural Language Processing and the 9th International Joint Conference on Natural Language Processing, EMNLP-IJCNLP 2019, Hong Kong, China, November 3-7, 2019, pages 540-551. Association for Computational Linguistics.

Mike Lewis, Yinhan Liu, Naman Goyal, Marjan Ghazvininejad, Abdelrahman Mohamed, Omer Levy, Veselin Stoyanov, and Luke Zettlemoyer. 2020. BART: denoising sequence-to-sequence pretraining for natural language generation, translation, and comprehension. In Proceedings of the 58th Annual Meeting of the Association for Computational Linguistics, ACL 2020, Online, July 5-10, 2020, pages 7871-7880. Association for Computational Linguistics.

Chin-Yew Lin. 2004. ROUGE: A package for automatic evaluation of summaries. In Text Summarization Branches Out, pages 74-81, Barcelona, Spain. Association for Computational Linguistics.

Yang Liu and Mirella Lapata. 2019. Text summarization with pretrained encoders. In Proceedings of the 2019 Conference on Empirical Methods in Natural Language Processing and the 9th International Joint Conference on Natural Language Processing (EMNLP-IJCNLP), pages 3730-3740, Hong Kong, China. Association for Computational Linguistics.

Yinhan Liu, Myle Ott, Naman Goyal, Jingfei Du, Mandar Joshi, Danqi Chen, Omer Levy, Mike Lewis, Luke Zettlemoyer, and Veselin Stoyanov. 2019. Roberta: A robustly optimized BERT pretraining approach. CoRR, abs/1907.11692.

Brett AS Martin, Bodo Lang, Stephanie Wong, and Brett AS Martin. 2003. Conclusion explicitness in advertising: The moderating role of need for cognition (nfc) and argument quality (aq) on persuasion. Journal of Advertising, 32(4):57-66.

Mani Maybury. 1999. Advances in automatic text summarization. MIT press.

Shashi Narayan, Shay B. Cohen, and Mirella Lapata. 2018. Don't give me the details, just the summary! topic-aware convolutional neural networks for extreme summarization. In Proceedings of the 2018 Conference on Empirical Methods in Natural Language Processing, pages 1797-1807, Brussels, Belgium. Association for Computational Linguistics.

Lawrence Page, Sergey Brin, Rajeev Motwani, and Terry Winograd. 1999. The pagerank citation ranking: Bringing order to the web. Technical report, Stanford InfoLab. 
Andreas Peldszus and Manfred Stede. 2015. Joint prediction in MST-style discourse parsing for argumentation mining. In Proceedings of the 2015 Conference on Empirical Methods in Natural Language Processing, pages 938-948. Association for Computational Linguistics.

Georgios Petasis and Vangelis Karkaletsis. 2016. Identifying argument components through textrank. In Proceedings of the Third Workshop on Argument Mining, hosted by the 54th Annual Meeting of the Association for Computational Linguistics, ArgMining@ACL 2016, August 12, Berlin, Germany.

Alec Radford, Jeffrey Wu, Rewon Child, David Luan, Dario Amodei, and Ilya Sutskever. 2019. Language models are unsupervised multitask learners. OpenAI blog, 1(8):9.

Sascha Rothe, Shashi Narayan, and Aliaksei Severyn. 2020. Leveraging pre-trained checkpoints for sequence generation tasks. Trans. Assoc. Comput. Linguistics, 8:264-280.

Victor Sanh, Lysandre Debut, Julien Chaumond, and Thomas Wolf. 2019. Distilbert, a distilled version of bert: smaller, faster, cheaper and lighter. arXiv preprint arXiv:1910.01108.

Benjamin Schiller, Johannes Daxenberger, and Iryna Gurevych. 2020. Aspect-controlled neural argument generation. CoRR, abs/2005.00084.

Sam Shleifer and Alexander M Rush. 2020. Pretrained summarization distillation. arXiv preprint arXiv:2010.13002.

Christian Stab and Iryna Gurevych. 2014. Identifying argumentative discourse structures in persuasive essays. In Proceedings of the 2014 Conference on Empirical Methods in Natural Language Processing (EMNLP), pages 46-56, Doha, Qatar. Association for Computational Linguistics.

Ilya Sutskever, Oriol Vinyals, and Quoc V. Le. 2014. Sequence to sequence learning with neural networks. In Advances in Neural Information Processing Systems 27: Annual Conference on Neural Information Processing Systems 2014, December 8-13 2014, Montreal, Quebec, Canada, pages 3104-3112.

Shahbaz Syed, Roxanne El Baff, Johannes Kiesel, Khalid Al Khatib, Benno Stein, and Martin Potthast 2020. News editorials: Towards summarizing long argumentative texts. In Proceedings of the 28th International Conference on Computational Linguistics, pages 5384-5396, Barcelona, Spain (Online). International Committee on Computational Linguistics.

Stephen E Toulmin. 2003. The uses of argument. Cambridge university press.

Teun A Van Dijk. 1995. Opinions and ideologies in editorials. In 4th International Symposium of Critical Discourse Analysis, Language, Social Life and Critical Thought, Athens, pages 14-16.
Henning Wachsmuth, Martin Potthast, Khalid AlKhatib, Yamen Ajjour, Jana Puschmann, Jiani Qu, Jonas Dorsch, Viorel Morari, Janek Bevendorff, and Benno Stein. 2017. Building an argument search engine for the web. In Proceedings of the 4th Workshop on Argument Mining, pages 49-59, Copenhagen, Denmark. Association for Computational Linguistics.

Douglas Walton, Christopher Reed, and Fabrizio Macagno. 2008. Argumentation Schemes. Cambridge University Press.

Lu Wang and Wang Ling. 2016. Neural network-based abstract generation for opinions and arguments. In Proceedings of the 2016 Conference of the North American Chapter of the Association for Computational Linguistics: Human Language Technologies, pages 47-57, San Diego, California. Association for Computational Linguistics.

Thomas Wolf, Lysandre Debut, Victor Sanh, Julien Chaumond, Clement Delangue, Anthony Moi, Pierric Cistac, Tim Rault, Remi Louf, Morgan Funtowicz, Joe Davison, Sam Shleifer, Patrick von Platen, Clara Ma, Yacine Jernite, Julien Plu, Canwen Xu, Teven Le Scao, Sylvain Gugger, Mariama Drame, Quentin Lhoest, and Alexander Rush. 2020. Transformers: State-of-the-art natural language processing. In Proceedings of the 2020 Conference on Empirical Methods in Natural Language Processing: System Demonstrations, pages 38-45, Online. Association for Computational Linguistics.

Haoyu Zhang, Jingjing Cai, Jianjun $\mathrm{Xu}$, and Ji Wang. 2019a. Pretraining-based natural language generation for text summarization. In Proceedings of the 23rd Conference on Computational Natural Language Learning (CoNLL), pages 789-797, Hong Kong, China. Association for Computational Linguistics.

Jingqing Zhang, Yao Zhao, Mohammad Saleh, and Peter J. Liu. 2020a. PEGASUS: pre-training with extracted gap-sentences for abstractive summarization. In Proceedings of the 37th International Conference on Machine Learning, ICML 2020, 13-18 July 2020, Virtual Event, volume 119 of Proceedings of Machine Learning Research, pages 11328-11339. PMLR.

Tianyi Zhang, Varsha Kishore, Felix Wu, Kilian Q. Weinberger, and Yoav Artzi. 2020b. Bertscore: Evaluating text generation with BERT. In 8th International Conference on Learning Representations, ICLR 2020, Addis Ababa, Ethiopia, April 26-30, 2020. OpenReview.net.

Yuan Zhang, Jason Baldridge, and Luheng He. 2019b. PAWS: Paraphrase adversaries from word scrambling. In Proceedings of the 2019 Conference of the North American Chapter of the Association for Computational Linguistics: Human Language Technologies, Volume 1 (Long and Short Papers), pages 1298-1308, Minneapolis, Minnesota. Association for Computational Linguistics. 